Supplemental Table 1. Characteristics of the study sample by diabetes onset group.

\begin{tabular}{lcccc}
\hline Characteristics* & $\begin{array}{c}\text { Age of Onset } \\
\mathbf{5 5 5} \text { years } \\
(\mathbf{n = 2 0 1})\end{array}$ & $\begin{array}{c}\text { Age of } \\
\text { Onset 55-64 } \\
\text { years } \\
(\mathbf{n = 2 5 5})\end{array}$ & $\begin{array}{c}\text { Age of Onset } \\
\mathbf{6 5 5} \text { years } \\
(\mathbf{n = 8 0 1})\end{array}$ & $\begin{array}{c}\text { Never } \\
\text { Diabetes } \\
(\mathbf{n = 4 5 1 4})\end{array}$ \\
\hline Examinations, n & $9.1 \pm 5.2$ & $11.8 \pm 6.4$ & $17.7 \pm 8.2$ & $12.8 \pm 6.6$ \\
Age at baseline, years & $40.6 \pm 7.4$ & $41.8 \pm 7.7$ & $42.0 \pm 8.2$ & $44.8 \pm 8.3$ \\
Age at death, years & $71.2 \pm 8.4$ & $75.2 \pm 8.3$ & $86.3 \pm 8.3$ & $79.2 \pm 10.4$ \\
Women (\%) & $93(46)$ & $99(39)$ & $434(54)$ & $2468(55)$ \\
Body mass index, kg/m ${ }^{2}$ & $28.8 \pm 5.9$ & $28.7 \pm 5.2$ & $26.0 \pm 4.4$ & $25.4 \pm 3.9$ \\
Current smoker $(\%)$ & $108(54)$ & $147(58)$ & $385(48)$ & $2393(53)$ \\
Fasting blood glucose, mg/dL & $169 \pm 72$ & $130 \pm 47$ & $102 \pm 15$ & $93 \pm 9$ \\
Non-fasting blood glucose, mg/dL & $127 \pm 54$ & $96 \pm 22$ & $88 \pm 18$ & $85 \pm 16$ \\
Cholesterol, mg/dL & $221 \pm 50$ & $221 \pm 44$ & $217 \pm 45$ & $223 \pm 44$ \\
HDL cholesterol, mg/dL & $42 \pm 14$ & $43 \pm 15$ & $48 \pm 16$ & $51 \pm 16$ \\
Hypertension $(\%)$ & $116(58)$ & $155(61)$ & $354(44)$ & $2204(49)$ \\
Use of anti-hypertensive therapy (\%) & $17(8)$ & $23(9)$ & $32(4)$ & $214(5)$ \\
Use of statins $(\%)$ & $2(1)$ & $5(2)$ & $4(0.5)$ & $23(1)$ \\
\hline
\end{tabular}

Data are shown as $\mathrm{n}(\%)$ or mean \pm SD.

Values displayed are from the first examination cycle where these were available, except for the age at death and the age of diabetes onset. 
Supplemental Table 2. Comparison of the disease duration between those who died from cardiovascular disease vs those who died from noncardiovascular death

Cardiovascular Deaths $\quad$ Coronary Deaths $\quad$ Non-cardiovascular Deaths

\begin{tabular}{|c|c|c|c|}
\hline Characteristic & $\begin{array}{c}\text { Mean duration of diabetes } \pm \text { SD } \\
\text { (No. Deaths) }\end{array}$ & $\begin{array}{c}\text { Mean duration of diabetes } \pm \text { SD } \\
\text { (No. Deaths) }\end{array}$ & $\begin{array}{c}\text { Mean duration of diabetes } \pm \text { SD } \\
\text { (No. Deaths) }\end{array}$ \\
\hline \multicolumn{4}{|c|}{ Age of diabetes onset } \\
\hline$<55$ years & $22.5 \pm 8.6(\mathrm{~N}=94)$ & $21.1 \pm 7.9(\mathrm{~N}=59)$ & $22.6 \pm 9.0(\mathrm{~N}=107)$ \\
\hline $55-64$ years & $13.4 \pm 7.7(\mathrm{~N}=114)$ & $11.5 \pm 7.2(\mathrm{~N}=66)$ & $16.2 \pm 9.0(\mathrm{~N}=141)$ \\
\hline$\geq 65$ years & $7.5 \pm 5.6(\mathrm{~N}=239)$ & $7.9 \pm 5.7(\mathrm{~N}=104)$ & $7.4 \pm 6 .(\mathrm{N}=562)$ \\
\hline Overall & $12.2 \pm 9.1(\mathrm{~N}=104)$ & $12.3 \pm 8.6(\mathrm{~N}=229)$ & $11.0 \pm 9.1(\mathrm{~N}=810)$ \\
\hline$P$-value & $\begin{array}{c}0.02 \text { (for cardiovascular deaths vs. } \\
\text { non-cardiovascular deaths comparison) }\end{array}$ & $\begin{array}{l}0.0004 \text { (for coronary deaths vs. non- } \\
\text { cardiovascular deaths comparison) }\end{array}$ & Reference \\
\hline
\end{tabular}




\section{Stroke Deaths}

No. Deaths

Odds Ratios (95\% CI)

\begin{tabular}{lcccc}
\hline $\begin{array}{l}\text { Characteristic } \\
\text { Age of diabetes onset }\end{array}$ & Unadjusted & Age of death and sex-adjusted & Multivariable adjusted* \\
$<55$ years & 5 & $3.49(1.36-9.01)$ & $3.24(1.23-8.55)$ & $2.15(0.35-13.17)$ \\
$55-64$ years & 6 & $3.18(1.33-7.61)$ & $3.18(1.32-7.65)$ & $2.74(0.69-10.84)$ \\
$\geq 65$ years & 8 & $1.06(0.50-2.28)$ & $1.17(0.53-2.56)$ & $1.05(0.43-2.56)$ \\
No diabetes & 42 & referent & referent & referent \\
$P$ trend & & $<0.0001$ & $<0.01$ & 0.29 \\
\hline
\end{tabular}

*Multivariable models are adjusted for age at death, sex, smoking status, systolic blood pressure, body mass index, and total cholesterol, use of antihypertensive medications, use of statins, and duration of diabetes (covariates are from first examination at which data were available). 
Supplemental Table 4. Odds ratio for cardiovascular versus non-cardiovascular deaths, adjusting for covariates assessed at the last examination.

\section{CVD Deaths}

CHD Deaths

Stroke Deaths

\begin{tabular}{|c|c|c|c|c|c|c|}
\hline Characteristic & No. Deaths & Odd Ratio (95\% CI) & No. Deaths & Odd Ratio (95\% CI) & No. Deaths & Odd Ratio (95\% CI) \\
\hline \multicolumn{7}{|c|}{ Age of diabetes onset } \\
\hline$<55$ years & 94 & $1.78(1.08-2.93)$ & 59 & $1.75(0.96-3.21)$ & 5 & $1.59(0.24-10.50)$ \\
\hline $55-64$ years & 114 & $1.59(1.10-2.31)$ & 66 & $1.56(1.00-2.43)$ & 6 & $1.78(0.43-7.29)$ \\
\hline$\geq 65$ years & 239 & $1.13(0.91-1.40)$ & 104 & $1.10(0.82-1.46)$ & 8 & $0.72(0.29-1.78)$ \\
\hline No diabetes & 1375 & Referent & 732 & Referent & 42 & Referent \\
\hline$P$ trend & & 0.013 & & 0.044 & & 0.481 \\
\hline
\end{tabular}

Multivariable models are adjusted for age at death, sex, smoking status, hypertension, body mass index, and total cholesterol, use of

antihypertensive, use of statins, and duration of diabetes (covariates are from the last examination, prior to death, at which data were available) 
Supplemental Table 5. Odds ratio for cardiovascular or coronary death (versus non-cardiovascular death) based on diabetes age of onset, adjusting for risk factors and socio-economic status.

\section{Cardiovascular Deaths}

No. Deaths

\section{Characteristic}

Age of diabetes onset

$$
<55 \text { years }
$$

55-64 years

$\geq 65$ years

Never diabetes

$P$ trend
1168
Odds Ratio (95\% CI)

Multivariable adjusted

$1.93(1.11-3.33)$,

$1.63(1.08-2.47)$

$1.14(0.91-1.43)$

referent

0.01

\section{Coronary Deaths}

Odds Ratio (95\% CI)

Multivariable adjusted

Multivariable models adjusted for age at death, sex, smoking status, systolic blood pressure, body mass index, total cholesterol, use of antihypertensives, use of statins, education, occupation and duration of diabetes, based on data from the first examination at which assessment data were available. 
Supplemental Table 6. Characteristics of the offspring participants according to parental diabetes-onset age

\section{Parental Diabetes Status}

\begin{tabular}{|c|c|c|c|c|}
\hline Characteristic & $\begin{array}{l}\text { Parents without Diabetes } \\
\qquad(\mathrm{n}=1720)\end{array}$ & $\begin{array}{c}\text { Late-Onset Diabetes in } \geq 1 \\
\text { Parent }(\mathrm{n}=321)\end{array}$ & $\begin{array}{c}\text { Early-Onset Diabetes in } 1 \\
\text { Parent* }(n=82)\end{array}$ & $P$ value \\
\hline Age, years & $33.8 \pm 9.5$ & $31.9 \pm 9.8$ & $33.8 \pm 8.6$ & 0.003 \\
\hline Women $(\%)$ & $882(51)$ & $154(48)$ & $36(44)$ & 0.26 \\
\hline Body mass index, $\mathrm{kg} / \mathrm{m}^{2}$ & $24.7 \pm 4.1$ & $25.3 \pm 4.9$ & $26.1 \pm 4.0$ & 0.001 \\
\hline Diastolic blood pressure, $\mathrm{mm} \mathrm{Hg}$ & $79 \pm 10$ & $79 \pm 11$ & $83 \pm 10$ & 0.006 \\
\hline Current smoker (\%) & $734(43)$ & $128(40)$ & $36(44)$ & 0.62 \\
\hline Total cholesterol, mg/dL & $195 \pm 38$ & $195 \pm 41$ & $197 \pm 33$ & 0.87 \\
\hline
\end{tabular}

Data are $\mathrm{n}(\%)$ or mean (SD). Abbreviations: HDL, high density lipoprotein. Characteristics are from the first examination at which data were available. Characteristics are from the first examination at which data were available.

*Including one early-onset only, or one early-onset plus one late-onset (there were no families with early-onset diabetes in both parents). 
Supplemental Table 7. Incident diabetes in offspring by diabetes age-of-onset in parents, according to offspring age bands.

\begin{tabular}{|c|c|c|c|c|c|}
\hline Age Band & Person-Years & Incident DM & No DM in Parents & Late $D M$ in $\geq 1$ Parents & Early DM in 1 Parent + \\
\hline$<55$ years & 41804.3 & 63 & & & \\
\hline Unadjusted model & & & 1.00 [referent] & $2.29(1.20-4.39)$ & $6.84(3.51-13.30)$ \\
\hline Multivariable-adjusted model* & & & 1.00 [referent] & $2.28(1.57-3.31)$ & $3.12(1.68-5.80)$ \\
\hline$\geq 55$ years & 26192.3 & 149 & & & \\
\hline Unadjusted model & & & 1.00 [referent] & $2.05(1.09-3.88)$ & $5.43(2.87-10.29)$ \\
\hline Multivariable-adjusted model* & & & 1.00 [referent] & $2.25(1.51-3.36)$ & $2.48(1.23-5.01)$ \\
\hline
\end{tabular}

Abbreviations: DM, diabetes.

*Adjusted for date of birth, sex, body mass index, smoking status

$\dagger$ Including 1 parent with early-onset diabetes or 1 with early-onset plus 1 with late-onset diabetes (there were no families with early-onset diabetes in both parents). 
Supplemental Table 8. Incident diabetes in offspring by diabetes age-of-onset in parents, with early-onset defined as age <50 years.

\begin{tabular}{|c|c|c|c|}
\hline & \multicolumn{3}{|c|}{ Parental Diabetes Status } \\
\hline & $\begin{array}{l}\text { Parents without Diabetes } \\
\qquad(\mathrm{n}=1715)\end{array}$ & $\begin{array}{c}\text { Late-Onset Diabetes in } \geq 1 \\
\text { Parents }(n=356)\end{array}$ & $\begin{array}{c}\text { Early-Onset Diabetes in } 1 \\
\text { Parent }(n=33) \dagger\end{array}$ \\
\hline No. with incident diabetes & 136 & 59 & 12 \\
\hline Person-years of follow-up & 55336 & 11255 & 956 \\
\hline Incidence per 1000 person-years $(95 \% \mathrm{CI})$ & $25(21,29)$ & $52(41,68)$ & $125(71,221)$ \\
\hline \multicolumn{4}{|l|}{ Hazard ratio $(95 \% \mathrm{CI})$} \\
\hline Unadjusted model & 1.00 [referent] & $2.20(1.56-3.11) \ddagger$ & $5.70(2.97-10.92) \ddagger$ \\
\hline Multivariable-adjusted model* ${ }^{*}$ & 1.00 [referent] & $2.23(1.55-3.21) \ddagger$ & $4.13(1.80-9.49) \ddagger$ \\
\hline
\end{tabular}

CI, confidence interval.

*Adjusted for age, sex, body mass index, and smoking status.

$\dagger$ Including 1 early-onset parent or 1 early-onset plus 1 late-onset parent.

$\ddagger P<0.001$ 